CAHIERS DE

NARRATOLOGIE

\section{Cahiers de Narratologie}

Analyse et théorie narratives

36 | 2019

Rhétorique et représentations de la culture mafieuse.

Images, rituels, mythes et symboles

\title{
Ritualisme, religion, codes symboliques des associations criminelles en milieu pénitentiaire
}

\section{Marco Soddu}

Translator. Manuela Bertone (de l'italien)

\section{OpenEdition}

\section{Journals}

Electronic version

URL: http://journals.openedition.org/narratologie/10022

ISSN: 1765-307X

Publisher

LIRCES

Electronic reference

Marco Soddu, «Ritualisme, religion, codes symboliques des associations criminelles en milieu pénitentiaire », Cahiers de Narratologie [Online], 36 | 2019, Online since 20 December 2019, connection on 20 December 2019. URL : http://journals.openedition.org/narratologie/10022

This text was automatically generated on 20 December 2019.

Article L.111-1 du Code de la propriété intellectuelle. 


\title{
Ritualisme, religion, codes symboliques des associations criminelles en milieu pénitentiaire
}

\author{
Marco Soddu
}

Translation : Manuela Bertone (de l'italien)

\section{Introduction}

1 Notre contribution fournit une évaluation détaillée d'actions criminelles spécifiques (définies par l'article 416 bis du Code pénal italien, consacré au délit d'association mafieuse) qui permettent au groupe criminel mafieux de communiquer au sein des lieux privatifs de liberté - mais également avec l'extérieur - sur des questions opératoires pour ses activités illicites et, partant, de développer des stratégies criminelles et de les appliquer.

2 Notre recherche découle d'une exigence fondamentale : la connaissance du phénomène mafieux, qui sans être une fin en soi, impose d'en analyser toutes les caractéristiques afin de mettre en place une action de prévention efficace et, au besoin, une action de répression systématique de tous les comportements illégaux planifiés, ordonnés et actés pendant la période de détention.

Compte tenu des limites tant spatiales que temporelles imposées à la communication carcérale, que la mafia doit contourner, notre objectif principal consiste à examiner les formes rituelles, symboliques et mythiques utilisées précisément dans le but de satisfaire les besoins primaires de la communication et de la diffusion d'informations. À ce propos, il convient de préciser que les échanges d'informations verbales de nature criminelle seront au cœur de notre analyse, et non les interactions quotidiennes normales (et par conséquent, anodines) entre détenus.

Nous ferons référence essentiellement aux coutumes carcérales de la 'Ndrangheta, la mafia calabraise, et de la Sacra Corona Unita (SCU), une sorte de dérivation de la mafia 
calabraise délimitée territorialement aux Pouilles, en particulier aux régions du Salento et de Brindisi.

Il est très important de noter que la SCU est née à l'intérieur d'un centre de détention, à l'initiative de Giuseppe Rogoli, entre le 24 et le 25 décembre 1981. Membre éminent du clan de la 'Ndrangheta Bellocco de Rosarno (Calabre), sous l'égide du capobastone ${ }^{1}$ Umberto Bellocco, Rogoli a créé la première ndrina des Pouilles dans la prison de Trani, où il était incarcéré. Rogoli est actuellement détenu: il purge trois peines d'emprisonnement à perpétuité.

Voici le serment de la SCU :

Je jure sur cette pointe de poignard baignée de sang d'être toujours fidèle à ce corps de société d'hommes libres, actifs et affirmant appartenir à la Sacra Corona Unita et de représenter partout son fondateur, Giuseppe Rogoli.

Et le serment de la 'Ndrangheta:

Bonsoir et une sainte soirée aux santisti ! Justement, en cette sainte soirée, dans le silence de la nuit et sous la lumière des étoiles et la splendeur de la Lune, je forme la chaîne sacrée! Au nom de Garibaldi, de Mazzini et de La Marmora, par des mots humbles, je forme la Sainte Société.

\section{L'affiliation en milieu pénitentiaire}

On peut certainement considérer la pratique de l'affiliation mafieuse comme le premier élément digne d'être signalé car il caractérise une pratique sédimentée et «institutionnalisée» au sein du contexte criminel. Le besoin constant d'agir clandestinement (même si diverses nuances et exceptions sont possibles) et de faire profil bas implique la nécessité d'un protocole communicatif basé essentiellement sur le langage non verbal, avec de forts éléments symboliques et rituels, associés à des connotations rappelant la dynamique religieuse.

9 Dans ce cadre, présentent un intérêt particulier les spécificités propres au contexte de la détention dans des établissements pénitentiaires où s'appliquent des régimes de haute sécurité (AS3 et 41 bis) ${ }^{2}$ et de surveillance conduisant à une limitation supplémentaire des possibilités de communication à connotation personnelle directe.

Avant l'affiliation au sein d'un centre de détention (rappelons qu'il s'agit toujours d'un contexte extrêmement contrôlé, où les systèmes de surveillance sont particulièrement efficaces), se met en place un curieux manège devant rester aussi secret que possible et que l'on peut considérer comme une sorte de manière mafieuse de faire sa cour.

11 Il est des individus spécialement attrayants, surtout aux yeux de la 'Ndrangheta et de sa consœur-dérivée la Sacra Corona Unita, généralement sous-estimée, non pas au niveau des enquêtes, des mises en examen et des condamnations, mais dans l'imaginaire collectif, et ce en dépit de la force dévastatrice dont cette dernière a fait preuve à certaines périodes, surtout dans les années 1980 et 1990.

12 Dans le passé récent, avant que ne s'affirme le quasi-monopole des clans calabrais sur la drogue venant de Colombie, le marché de l'héroïne d'abord, le marché de la cocaïne ensuite, ont eu besoin d'individus fort charismatiques, si possible déjà en possession de «contacts » utiles en vue de l'approvisionnement. Or, la prison a souvent été le lieu idéal où des individus non liés aux clans locaux, mais en possession desdits contacts, ont pu être repérés et recrutés. Pour les mafieux, la question s'est posée de savoir comment traiter avec ces individus afin d'exploiter leurs « connaissances » et d'accéder 
$\mathrm{au}$ « canal ». Suite à une demande directe de partage avec l'individu faisant fonction de référent sur une fourniture de drogue et en cas de refus de collaboration de la part de ce dernier, des pratiques modérées d'intimidation ont été mises en place, visant à le faire apparaître comme un « infâme » aux yeux de toute la population carcérale. En cas de non coopération, on a pu aller jusqu'au meurtre. L'expression typique utilisée pour signaler négativement l'individu réticent est toujours la même : « lui, il n'est pas bien, c'est un espion!».

13 N'oublions pas que la vidéosurveillance à l'intérieur des structures carcérales est relativement récente et que l'histoire pénale foisonne de cas de meurtres à l'intérieur des prisons : songeons par exemple aux révoltes dans les établissements de détention, qui ont vu l'émergence des soi-disant killers des prisons.

14 Les prisons ont toujours été le principal lieu d'affiliation, à tous les niveaux. Le rituel d'affiliation se déroulait généralement dans la cour réservée à la promenade et quasiment jamais dans la cellule: le signe évident de cette pratique est à ce jour l'allumage simultané de cigarettes par des individus regroupés en un cercle où même les non fumeurs allument une cigarette. La cigarette est utilisée pour brûler l'image pieuse du saint (partie spécifique du rituel) et aussi pour transmettre un signal très clair aux individus étrangers au rituel afin qu'ils ne s'approchent pas. Un autre message est également envoyé dans ce cadre : il y a un nouvel affilié, à qui on doit désormais s'adresser en l'appelant compare (compagnon) et qui, à son tour, s'adressera à chacun en utilisant ce même mot.

Mais comment peut-on devenir un affilié ? Fondamentalement, deux cas de figure sont possibles. Dans le premier, un boss détenu exprime sa volonté de recruter un individu qu'il désigne en utilisant l'expression "c'est un type bien!», en raison d'antécédents criminels notoires et/ou de capacités avérées, parfois sans qu'une " mise à l'épreuve » soit nécessaire. Rappelons que, pour la bonne forme, la dote (la dot, c'est-à-dire l'adhésion au clan mafieux, avec un grade dans la hiérarchie) doit être demandée directement par le futur affilié et non par des membres déjà rattachés à l'association, même si concrètement la demande peut être formulée de manière «indirecte ", avec l'intercession de tierces personnes. Si - cas rarissime - un individu refuse l'affiliation, la sentence prononcée par le boss est sans appel: «dehors, tu ne pourras plus rien faire !». Aux mots s'ajoutent les pratiques d'intimidation que nous avons déjà évoquées et qui peuvent aller jusqu'à la mise à mort du rétif.

16 Généralement, c'est un détenu qui se présente au chef mafieux pour lui soumettre sa demande d'affiliation au clan. Dans ce cas, l'individu peut être soumis à une procédure d'« évaluation sur le terrain » : sur ordre du boss, on lui demande d'exercer un acte de violence permettant de vérifier ses capacités et son niveau de soumission (que l'on veut total) en tant qu' « apprenti affilié ».

\section{Affiliation et religion : deux pratiques en regard}

17 Le rituel d'affiliation a lieu uniquement et exclusivement le samedi, qui est aussi le jour réservé au "partage des spartenze», à savoir les cigarettes que l'on distribue aux membres du groupe criminel. Le samedi est communément considéré un jour saint et c'est justement en ce jour saint que se noue le lien entre un parrain et un nouvel affilié. Le renvoi à la sphère religieuse et à l'univers maçonnique est constant et manifeste (il est évident dans le texte du serment, que nous avons présenté ci-dessus). Le jeudi 
(supposé être le jeudi saint issu de la pratique religieuse) est le jour où « l'on transmet la nouvelle " d'une cellule à l'autre, c'est-à-dire où l'on annonce officiellement l'identité des nouveaux affiliés du samedi précédent, que tous les membres de l'association mafieuse devront obligatoirement saluer et embrasser.

Une autre référence à la religion est visible dans le rituel dit «baptême » des locaux de rétention. Il s'agit de lieux institutionnels considérés comme « impurs " (autant que les casernes des carabiniers et, de manière générale, l'ensemble du dispositif policier), des lieux qu'il faut donc soumettre à un rite de "purification " pouvant les priver de leur signification première, de leur destination naturelle et, en langage mafieux, les "rendre honorables». Cette procédure est fortement révélatrice de la conception mafieuse de l'anti-État, du rejet total des institutions nationales et de la volonté de créer un état mafieux dans l'État de droit (un État que l'on voudrait rayer de la carte, même si on en admet l'existence sur un plan formel).

L'aspect religieux, nous l'avons dit, est constamment présent dans les pratiques rituelles, dans l'usage d'images pieuses, dans le choix des jours consacrés aux affiliations. Il convient de préciser que la possession d'images pieuses est permise dans les prisons italiennes, car une éventuelle interdiction porterait atteinte au droit à la pratique religieuse reconnu à tout détenu. De toute évidence, la tradition, en particulier la tradition religieuse, fait l'objet d'un détournement à la fois à des fins rituelles et en tant que moyen d'auto-légitimation. Il est indéniable que l'exhibition surfaite du rituel usurpé, la mystification des aspects mythiques du fait religieux sont utilisées pour "régulariser" les pratiques illicites et, partant, l'association mafieuse elle-même.

20 Notons enfin que dans le cadre du serment, qui concerne le niveau le moins élevée de la hiérarchie, la santa, comme les plus hauts niveaux, on utilise également un comprimé (élément terrestre, certes, mais riche en connotations sacrificielles), symbolisant le cyanure (non disponible dans les prisons) que l'affilié pourrait être amené à avaler un jour pour éviter de trahir les siens, sous la menace policière par exemple.

\section{Éléments symboliques}

21 Le symbole est également un élément fondamental de la communication mafieuse. Mélangés aux aspects rituels, les éléments symboliques se manifestent dans les circonstances en apparence les plus simples, qui, si l'on regarde de près, s'avèrent être plutôt complexes. Songeons à l'échange de salutations : la poignée de main, avec le positionnement du pouce à l'intérieur de la main du récepteur identifie un sgarro (niveau intermédiaire des sphères mineures); le sourcil caressé indique que l'on est en présence d'un trequartino (niveau intermédiaire des hautes sphères); le menton frotté signifie que l'on a affaire à une santa.

$\mathrm{Au}$ sein d'une structure de détention, la possibilité de se procurer des objets interdits, notamment des armes, a toujours été, par la force des choses, absolument capitale pour les membres des groupes mafieux. Les symboles jouent un rôle essentiel dès lors qu'il s'agit de remplacer les armes par des objets légaux et d'usage quotidien : une simple fourchette représente un pistolet, un coupe-ongles ouvert fait fonction de poignard, tandis qu'une aiguille symbolise le couteau classique qui permet de piquer et de faire gicler le sang pendant le processus d'affiliation. 
analyse similaire peut être faite pour les cartes, qui ont toujours été primordiales dans le rituel en raison de leur pouvoir symbolique. Ainsi, l'as de cœurs représente l'« infâme ", l'as de piques le capobastone (souvent tatoué avec ce même symbole) et l'as de carreaux un affilié. Les cartes basses (généralement les 3, 4 et 5) symbolisent les hommes de main ; la dame symbolise le soldat sans grade et le valet, le « caporal ».

cela constitue de ce qu'on appelle la smazzettata, une pratique typique de la plus haute importance et digne de notre attention, puisqu'elle est gérée et mise à exécution par un sgarro ou une santa. L'individu préposé au jeu de cartes peut aussi utiliser une carte pour se présenter sans prononcer un seul mot : avec un as de piques il signale être le capo della società (chef de la confrérie), avec un sept de carreaux il se présente comme santista, avec le trois de piques, comme sgarrista. Montrer un quatre de cœur sert à signaler la présence d'un « infâme » et à transmettre tacitement un message clair pour tous les affiliés : l'infâme doit être "vidé ", à savoir battu (parfois à mort), pour qu'on l'évacue vers un autre secteur de détention.

S'agissant d'actions violentes et de règlements de comptes, on peut remarquer que les agressions menées à travers les barreaux blindés des portes des cellules sont désormais très rares depuis qu'un un boss battu par un autre boss justement à travers les barreaux de sa cellule a décrété qu'il s'agissait là d'une « mauvaise chose ».

\section{Tatouages et marques sur la peau}

Le tatouage de l'as de piques utilisé pour identifier un capobastone était autrefois accompagné du tatouage d'une rose sous l'aisselle gauche. Depuis quelques années, la rose est tombée en désuétude: trop courante, elle avait perdu sa fonction initiale consistant à établir immédiatement l'appartenance d'un individu à l'organisation criminelle ainsi que son grade.

Le tatouage sur le dos représentant saint Michel archange, le patron de la 'Ndrangheta, est très répandu, tout comme l'image stylisée de la prison de Favignana qui, selon une légende, rappelle l'épopée des trois chevaliers venus d'Espagne après avoir commis un crime d'honneur, qui ont ensuite fondé en Sicile, en Campanie et en Calabre les trois mafias «historiques». À l'instar de l'élément religieux, le mythe, le symbole et le tatouage sont parfois présents dans une seule et même image.

Parmi les motifs récurrents dans les dessins tatoués, on remarque les deux lions liés par une chaîne de vingt-quatre anneaux avec un anneau supplémentaire décroché du reste, qui génèrent le chiffre vingt-cinq, symbolisant la Noël, à savoir un élément foncièrement religieux rappelant la naissance de Jésus-Christ.

Les squelettes stylisés, empoignant un poignard de la main droite, un cercueil placé à côté des pieds, sont également fréquents : devant le cercueil, on trouve habituellement un livre où sont dessinés une étoile et un fer de cheval et où sont gravés trois poignards croisés pouvant être accompagnés d'un calice et d'un crâne sur lequel est posée une bougie allumée.

Le livre représente l'histoire et la tradition de la 'Ndrangheta, afin de souligner la durée séculaire de cette organisation criminelle, tandis que les tatouages relatifs à des événements sanglants relatent les épisodes ayant entraîné la mort auxquels l'individu tatoué a pu être associé. Les tatouages sans références à la violence relèvent du pur symbolisme et des mythes abstraits propres de la 'Ndrangheta.

Cahiers de Narratologie, 36 | 2019 
31 L'étoile tatouée représente la copiata, c'est-à-dire la réunion des cinq personnes ayant participé au rituel baptismal nécessaire à l'affiliation. Le fer de cheval est le symbole de la confrérie et fait référence au groupe qui assiste au rituel d'initiation mafieux. Le cercle a une double signification. D'une part, la protection accordée aux personnes appartenant au groupe criminel, de l'autre, une sorte d'encerclement et de contrôle sans solution de continuité, prémonitoire de ce qui pourrait arriver à ceux qui trahissent et du fait qu'il n'y aura pas d'issue.

Parmi les signes corporels ayant survécu au fil du temps, on trouve les croix gravées dans le dos à l'aide de couteaux ou de lames de rasoir. Il s'agit d'une sorte de marque ayant pour but d'identifier différents niveaux et grades au sein de l'organisation criminelle : trois croix identifient un diritto; deux croix, un trequartino; une croix, un vangelo. Les croix peuvent aussi être gravées les unes sur les autres. En revanche, lorsqu'elle est tatouée sur le pouce de la main, la croix appartient à un sgarro. Un point tatoué sur l'index fait référence au grade de picciotto-camorrista.

\section{Langage verbal et langage non verbal}

Une réflexion s'impose sur le fait que la pratique mafieuse, commune à toutes les associations, interdit formellement de montrer son appartenance, tandis que dans la pratique quotidienne il en va tout autrement. Dans le cadre d'un affrontement physique entre deux affiliés, par exemple, la "déclaration » précède l'assaut: chacun déclare à quel groupe il appartient et fait état de son rang; et cela, souvent, en présence de l'ensemble de la population carcérale.

En revanche, lorsqu'un individu extérieur à l'association mafieuse commet une action digne de punition, s'il est connu d'un membre de l'organisation, il pourra être racheté par ce dernier, qui intervient pour son propre intérêt ou dans l'intérêt de l'association elle-même, et qui s'engage à le punir personnellement (généralement en le frappant en public). Le membre de l'organisation devient de ce fait responsable en cas de récidive, sachant que la récidive n'est absolument pas acceptée.

Concrètement, un mafieux se trouve toujours en équilibre précaire entre l'impératif de l'anonymat et la nécessité de faire connaître son rang pour mener des activités criminelles. À l'intérieur d'un établissement de détention, le silence total ne permettrait d'exercer aucun pouvoir, puisqu'un boss ni connu ni reconnu ne pourrait recréer en milieu carcéral l'habitat criminel dont il dispose à l'extérieur pour ses activités illicites, sachant que la situation de détention constitue déjà en elle-même une entrave de taille.

37 Le rituel du primo ingresso (première entrée) en cellule est révélateur. Le dialogue est structuré comme suit :

- «Puis-je entrer?»

- «Allez-y, entrez!»

- «Allez-y, entrez : c'est ce qu'on dit aux chevaux. Pour la première, la deuxième et

la troisième fois, je ne vois ni de porte d'entrée, ni de fenêtre de sortie et je vous

demande, puis-je entrer?»

- « Je vous en prie, installez-vous!» 
- Le temps de promenade est limité, par rapport aux détenus de droit commun, à deux heures par jour et s'effectue en régime d'isolement.

- Le détenu est constamment surveillé par une unité spéciale de la police pénitentiaire (GOM - Gruppo Operativo Mobile) qui, à son tour, n'entre pas en contact avec d'autres agents de la police pénitentiaire.

- Les visites au parloir de membres de la famille sont limitées, tant au niveau quantitatif (une visite d'une heure par mois maximum) qu'au niveau qualitatif (une haute cloison vitrée empêche tout contact physique). Une conversation téléphonique mensuelle d'une durée maximale de dix minutes avec des membres de la famille et des conjoints peut être autorisée, par décision motivée du directeur de d'établissement, uniquement pour les détenus qui n'ont pas bénéficié de visites.

- Les entretiens du détenu avec l'avocat assurant sa défense ne sont limités ni en nombre ni en durée.

- Tout courrier entrant et sortant ne peut être acheminé sans avoir obtenu un « visa » de contrôle.

- Limitation des sommes d'argent et des objets en possession du détenu dans sa cellule (stylos, cahiers, bouteilles, etc.) ainsi que des objets pouvant lui parvenir de l'extérieur. - Exclusion des instances de représentation des détenus et des internés.

\section{Conclusion}

Les pratiques que nous avons examinées, à commencer par le serment (riche en éléments maçonniques), en particulier dans l'univers de la 'Ndrangheta, sont caractérisées par d'indiscutables éléments rituels, symboliques et religieux. L'origine de ces pratiques est ancienne. Désormais, elles sont surtout transmises sous forme orale, mais des textes codés consacrés aux rituels d'affiliation à la 'Ndrangheta existent qui ont récemment été découverts par les forces de police et versés dans des dossiers 
d'enquête. Il apparaît que ces textes ont été modifiés et adaptés à cause des interdictions prévues par les Règles pénitentiaires italiennes que nous avons évoquées à plusieurs reprises (Loi $\mathrm{n}^{\circ} 354$ du 26 juillet 1975). Qui plus est, la modernisation des systèmes de vidéosurveillance, visibles et connus de tous en prison, a poussé les détenus à privilégier la communication discrète. À l'extérieur des établissements pénitentiaires, la crainte d'être interceptés et décryptés, a conduit les chefs mafieux à adopter des stratégies de communication semblables à celles que l'on pratique dans les centres de détention. Autrement dit, dans la communication verbale entre individus non placés en détention, on remarque des caractéristiques de la communication non verbale typique du milieu pénitentiaire. Les prisons, quant à elles, sont en passe de devenir des sortes de «laboratoires linguistiques» où l'on développe et l'on teste l'interaction criminelle codifiée, dans le but d'en assurer aussi le rayonnement à l'extérieur.

Comme nous l'avons précisé dans l'introduction, l'objectif de notre recherche est de fournir une série d'indications et d'informations permettant de mieux contrôler la communication criminelle et, surtout, d'éviter qu'elle puisse avoir lieu à l'intérieur comme à l'extérieur des prisons, y compris lorsque les détenus sont soumis au contrôle direct de l'État. De toute évidence, il est souhaitable de limiter encore plus la communication et surtout de restreindre davantage les activités partagées, en cellule, lors de la promenade et en général dans tous les espaces communs.

En outre, il est opportun de réfléchir sur les influences réciproques entre associations mafieuses: si les détenus étaient divisés par groupes d'appartenance mafieuse, ils pourraient communiquer plus simplement, mais cela limiterait l'acquisition de nouveaux contacts criminels dans la section de détention; en revanche, si les détenus condamnés pour association mafieuse étaient répartis selon un critère d'hétérogénéité criminelle, cela conduirait à l'acquisition de nouvelles perspectives criminelles (y compris en termes de diversification et d'opportunité criminelle), mais aurait pour effet externe positif la diminution du potentiel organisationnel et opérationnel de chaque clan.

L'hypothèse de créer des circuits « mixtes ", partagés entre détenus mafieux et détenus de droit commun, doit être résolument écartée, puisque le passé nous a montré sans équivoque à quel point le mélange n'a fait que conduire à l'augmentation exponentielle des affiliations de détenus de droit commun.

L'élimination totale des échanges mafieux, bien sûr, n'est pas du tout facile à réaliser (surtout à court terme), étant donné que, comme nous l'avons dit, il existe toute une série de « consignes » criminelles qui, pratiquement, sont données sans prononcer un seul mot.

Dans ce domaine, il serait utile de mettre en place un échange permanent d'informations entre appareils de l'État, surtout par le biais de la rédaction de " fiches " relatives aux contacts noués par les mafieux en prison, en particulier avec les représentants d'autres groupes criminels. Il faudrait ensuite se focaliser, au terme de la période d'incarcération, sur la mise en place de contrôles ciblés ayant pour objet leurs éventuelles activités illicites ultérieures. Cela permettrait, par ailleurs, de se procurer des renseignements, y compris préventifs, concernant leurs projets et mouvements criminels, vraisemblablement conçus dans le cadre de nouvelles alliances nouées en prison. 
mbreux résultats encourageants ont été obtenus grâce à l'application du régime de détention 41 bis et on pourrait souhaiter que des points spécifiques de cette norme s'appliquent également aux AS3, mais il est indéniable que la stratégie de prévention et de défense n'est pas parmi les plus simples à programmer et à appliquer, étant donné l'énorme complexité (non seulement juridique, mais aussi historique, culturelle et sociale) de la matière en objet.

\section{NOTES}

1. Au sein de la 'Ndrangheta, capobastone est le nom donné à celui qui dirige une ndrina, à savoir un groupuscule local généralement formé autour d'une famille (NdT).

2. «AS » (Alta Sicurezza) correspond à la section de haute sécurité, le niveau AS3 étant réservé aux détenus mafieux ayant dirigé un trafic de drogue. L'article 41 bis du Code de procédure pénale italien prévoit un régime carcéral spécial pour les chefs de la mafia $(N d T)$.

\section{ABSTRACTS}

L'articolo nasce dall'esigenza di comprendere le peculiarità del fenomeno mafioso, con un interesse specifico rivolto alle dinamiche di matrice comunicativa. Si analizzano i ritualismi, vagliando l'insieme di pratiche che consentono una modalità comunicativa all'interno di luoghi dove l'interazione è rigidamente controllata, come gli Istituti Penitenziari. Lo scambio informativo all'interno di strutture a connotazione protetta è infatti un focus imprescindibile, visto il continuo passaggio di informazioni che avviene attraverso l'utilizzo di rituali, simboli e miti. Nelle carceri si costituisce quindi un simil-nucleo sociale che, con le dovute proporzioni, ritrae quello presente all'esterno, fatte salve le limitazioni colloquiali da aggirare. Infatti, nonostante i severi divieti imposti, le mafie sono riuscite a elaborare validi succedanei dei loro abituali processi esterni che hanno reso possibile, in ambito intramurario, l'implementazione di pratiche apparentemente impensabili (affiliazioni, estorsioni, omicidi e spaccio di droga). I modelli osservabili delle fattezze comunicative di riferimento sono eterogenei: si va dall'affiliazione, con connotazioni cavalleresche, massoniche e carbonare, alla cultura definibile come mafiosa, rintracciabile nei social media, nel cinema, nella musica e anche nel ruolo simbolico che hanno i murales e ancora di più i tatuaggi.

Cet article répond à la nécessité de comprendre les spécificités du phénomène mafieux et se consacre en particulier aux dynamiques de la communication. Les rituels sont analysés ainsi que l'ensemble des pratiques qui permettent de communiquer dans des lieux où l'interaction est strictement contrôlée, c'est-à-dire les établissements pénitentiaires. Il est essentiel de se focaliser sur l'échange d'informations au sein de ces structures protégées, puisqu'un réseau continu d'interactions y est activé par l'utilisation de rituels, mythes et symboles. Bien qu'il faille 
contourner un certain nombre d'obstacles communicatifs, une sorte de tissu social se forme dans les prisons, qui, toutes proportions gardées, reproduit la structure sociale existante à l'extérieur. En effet, malgré les sévères interdictions imposées par la loi, les mafias ont réussi à créer des ersatz valables de leurs procédés habituels, mettant ainsi en place des pratiques normalement impensables en milieu carcéral (affiliations, extorsions, homicides, trafic de drogue). S'agissant de communication, les modèles de référence que l'on peut observer sont hétérogènes: de l'affiliation, renvoyant aux pratiques chevaleresques, maçonniques ou des carbonari, à la culture à proprement parler mafieuse, que l'on peut repérer dans les médias sociaux, au cinéma, dans la musique autant que dans le rôle symbolique joué par les graffitis et plus encore par les tatouages.

\section{INDEX}

Chronological index: XXe siècle, XXIe siècle, années 1970

Mots-clés: affiliation, tatouages, symboles, rituels, codes, franc-maçonnerie, communication, régime carcéral

Geographical index: Italie

\section{AUTHORS}

\section{MARCO SODDU}

Marco Soddu est expert criminologue auprès du ministère italien de la Justice. Il a obtenu un Ph.D. en History \& Archives à l'University College Dublin suite à un Master en criminologie et une Laurea en sciences politiques à l'Université de Cagliari. Il a été Visiting Researcher auprès du John Jay College of Criminal Justice de New York, de la JFK Library (Boston), de Toronto University, Corvinus University (Budapest) et University College Dublin. Ses recherches et ses activités professionnelles sont consacrées notamment au terrorisme international, au profilage criminel, à la cybercriminalité, à la cybersécurité, à l'évaluation des risques de récidive et à la prévention situationnelle des crimes. Parmi ses publications récentes, on peut citer l'ouvrage Terrorismo, pericolosità sociale e recidiva (2016) et les articles parus dans Brainfactor : «Il profilo del riconvertito in ambito detentivo - Criticità e prospettive di intervento » (2018), « L'Imam e la pratica Islamica in ambito carcerario. Una proposta operativa contro la radicalizzazione » (2018), «Prisonizzazione e reclutamento terroristico » (2017). 